\title{
All-polarization maintaining fiber laser and pulse compressor
}

\author{
Bo Xu, Amos Martinez, Sze.Yun. Set, and Shinji Yamashita
}

\begin{abstract}
Fiber lasers have become one of the most important technologies in various industrial, medical and scientific applications. However, their vulnerability to environmental disturbances, such as vibrations and thermal fluctuations, limits the range of practical operations outside of the laboratory. In this paper, we report a simplified, all-normal dispersion, completely all-polarization-maintaining fiber integrated laser operating at $1030 \mathrm{~nm}$. Our laser system employs a single pump laser diode and an all-polarization maintaining fiber pulse compressor capable of generating 700 fs pulses with a pulse energy of $0.45 \mathrm{~nJ}$.
\end{abstract}

Index Terms - Fiber lasers, Laser mode locking.

\section{INTRODUCTION}

$\mathrm{T}$ HE recent advances in ultrafast fiber laser technologies have led to a steep growth in their deployment for a range of applications including spectroscopy [1], optical imaging [2] and material processing [3]. From the point of view of industrial applications, fiber laser systems, unlike solid-sate laser system, provide advantages such as compactness, cost effectiveness, passive cooling and turn-key operability in an alignment-free configuration. Furthermore, for many applications, environmental stability is a critical requirement. Thus, commercial fiber laser systems must be impervious to external perturbations, such as thermal fluctuations and mechanical vibrations. In fiber-based systems, including fiber lasers, birefringence can be a key destabilizing factor, which could potentially lead to the deterioration of system performance. It has been proposed that the Faraday rotator mirror could compensate birefringence of the photonic crystal fiber (PCF) used for the intra-cavity dispersion compensation in ytterbium $(\mathrm{Yb})$ doped fiber laser [4,5]. In addition, the residual birefringence from PCF was employed to act as the

Manuscript received XXXX, 2018.

Bo Xu is with the Department of Engineering Science, Graduate School of Informatics, The University of Electro-Communications (UEC), 1-5-1 Chofugaoka, Chofu, Tokyo 182-8585, Japan (e-mail: xubo57@ uec.ac.jp).

Amos Martinez was with Aston Institute of Photonic Technologies, Aston University, Aston Triangle, Birmingham B4 7ET, UK*Currently with Toshiba Research Europe Ltd., 208 Cambridge Science Park, Milton Road, Cambridge, CB4 0GZ, UK (e-mail: amos.martinez@crl.toshiba.co.uk).

Sze.Yun.Set is with Research Center of Advanced Science and Technology, The University of Tokyo,4-6-1 Komaba, Meguro-ku, Tokyo, 153-8904, Japan (e-mail: set@cntp.t.u-tokyo.ac.jp).

Shinji Yamashita is Research Center of Advanced Science and Technology, The University of Tokyo,4-6-1 Komaba, Meguro-ku, Tokyo, 153-8904, Japan (e-mail: syama@cntp.t.u-tokyo.ac.jp).

* The work was done when the first author Bo Xu worked in Research Center of Advanced Science and Technology, The University of Tokyo. intracavity Lyot filter to initiate mode-locking [6]. Nevertheless, they still suffered from other issues, such as limited pulse energy and the inability to be completely integrated in all-fiber format inclusive of the pulse compressor. It is well known that the negative and often unpredictable impact of fiber birefringence can be managed by using polarization maintaining (PM) fiber and components. On the other hand, there has been an increasing interest and improved understanding of mode-locking mechanisms such as stretched-pulse mode-locking [7], self-similar pulsing [8], and dissipative solitons [9]. These result in significant performance improvements in fiber laser technology and a fascinating evolution striving towards higher powers, shorter pulses and improved stability.

Passively mode-locked fiber lasers are amongst the best pulsed sources available today as they can produce transform-limited femtosecond pulse trains with a simple cavity structure without the need for active components such as intensity modulators. In practice, they are typically realized by utilizing a nonlinear intensity-dependent component called a saturable absorber (SA). Semiconductor saturable absorption mirrors (SESAMs) have been widely incorporated in all-PM fiber laser configurations [10,11]. Emergence of the pigtailed SESAM realizes the compatibility to an all-fiber configuration, however, there is still room for improvement such as the operating lifetime [12]. The nonlinear polarization evolution (NPE), an excellent mode-locking technique which utilizes the Kerr effects in optical fiber, provides a fast artificial saturable absorption effect with a deep and adjustable modulation depth $[13,14]$. Nevertheless, in most cases, NPE mode-locking is typically implemented with bulk free-space optics that can suffer from long term reliability and reproducibility issues due to external environmental perturbations. Furthermore, NPE often requires realignment of the laser cavity polarization states from time to time. Recently, an NPE-based femtosecond mode-locked laser has been reported with an all-PM fiber cavity. However, it is still sensitive to mild mechanical stresses due to the short beat length of the PM fibers [15].

An alternative method to achieve stable mode-locking operation in an all-PM-fiber configuration is to employ the nonlinear amplifying loop mirror (NALM) as the mode locker. The NALM, proposed in the late 1980s, employs a beam splitter that separates the incoming beam into two counter propagating components that reach the gain fiber and are amplified at different times, offset by a piece of optical fiber for nonlinear phase-shift generation, see Fig.1. Due to the 
imbalance of the power between the two counter-propagating beams passing through the nonlinear fiber, they will accumulate different amount of nonlinear Kerr phase shifts. When these counter-propagating beams interference back at the splitter, it will create the effect of a fast, artificial saturable absorption which favors a pulsed operation over a continuous wave $(\mathrm{CW})$ operation [16,17]. Recently, there is a growing interest in using NALMs in all-PM-fiber configuration with PM fiber compatibility [18-27], particularly the Yb-doped NALM-based fiber lasers, primarily because of their promising operating wavelength for practically industrial applications and their power scalability. However, in these previously reported Yb fiber oscillators, multiple laser diodes (LDs) are employed, and extra-cavity free-space pulse pulse compressors are typically required, which give negative impacts in terms of cost effectiveness, system complexity and robustness [20,21,23-25].

Here, we propose and demonstrate a simple, compact, all-PM fiber, all-normal-dispersion pulsed fiber laser emitting at $1030 \mathrm{~nm}$. This elegant $\mathrm{Yb}$-doped oscillator configuration employs only a single LD, and the output pulses are compressed using an all-PM-fiber pulse compressor to generate a transform limit pulses with a pulse width of $700 \mathrm{fs}$ and a pulse energy of $0.45 \mathrm{~nJ}$. The compactness and simplicity of this proposed laser makes it suitable for a range of applications requiring environmental stability and robustness.

\section{ALL-FiBER POLARIZATION-MAINTAINING OSCILLATOR}

Figure 1 shows the layout of the proposed all-fiber oscillator and compressor system. It has the figure-of- 8 configuration and incorporates a NALM as the mode-locker. In the active NALM section (left part), a lowly-doped ytterbium fiber (core absorption: $80 \mathrm{~dB} / \mathrm{m}$ at $975 \mathrm{~nm}$, PM-YSF-LO, Nufern) was forward-pumped by a $976 \mathrm{~nm}$ diode laser through a 980/1030 PM-wavelength division multiplexer (WDM). A $2.5 \mathrm{~m}$ length of PM-980 fiber is used to provide the asymmetric nonlinear phase shift between the lights in clockwise and counterclockwise directions within the NALM. Additionally, an in-line polarizer is employed to enhance the polarization extinction ratio (PER) as well as the mode-locking stability. The main loop consists of an optical isolator (ISO) to ensure a unidirectional laser operation. An optical bandpass filter with a $2.8 \mathrm{~nm}$ spectral half-width is employed to reduce the large positive-chirp and to limit the spectral bandwidth of the pulses which are relaunched back into NALM. A 50:50 splitter couples the NALM to the main laser cavity and the laser output is tapped through the 20\% port as the output of a 20:80 splitter. The threshold pump power for initiating the mode-locking operation is approximately $230 \mathrm{~mW}$. Once the mode-locked operation is established, a stable pulsing operation could be maintained over a wide range of pump powers (from $100 \mathrm{~mW}$ to $300 \mathrm{~mW}$ ). The spectral profile depicted in Figure 2 (a) show the characteristic spectral shape of an all normal dispersion fiber laser with a full-width-half-maximum (FWHM) spectral bandwidth of $3.1 \mathrm{~nm}$, centered at $1030 \mathrm{~nm}$. We tested the stability of optical spectrum operating at different temperature settings. As plotted in Fig. 2(a), the output spectrum remained the same over a temperature variation of 10 degrees from $25^{\circ} \mathrm{C}$ $-35^{\circ} \mathrm{C}$. The output spectrum was only modestly distorted when the testing temperature was set at relatively high at $40^{\circ} \mathrm{C}$. Average output power was about $5 \mathrm{~mW}$, corresponding to a pulse energy of $0.45 \mathrm{~nJ}$. The laser operates at its fundamental repetition rate at a frequency of $12 \mathrm{MHz}$. The stability of the laser was investigated from the RF spectrum which was measured using an electrical spectrum analyzer with a span of 5 $\mathrm{kHz}$, and resolution bandwidth of $30 \mathrm{~Hz}$. The RF signal-to-noise ratio (SNR) of the laser was measured to be 90 $\mathrm{dB}$ at the fundamental frequency of $12 \mathrm{MHz}$, as shown in Fig. 2 (b) and $\sim 70 \mathrm{~dB}$ at the $20^{\text {th }}$-order frequency of $240 \mathrm{MHz}$ in inset of Fig.2(b). In addition, it showed the stability of the output spectrum as the inset of Fig. 2(a). Meanwhile, single-pulse operation was verified with the oscilloscope waveform as shown in Fig. 2(c).

The key feature of such all-normal dispersion lasers is the linearly chirped property of the output pulse [9]. The output pulses were characterized using a frequency-resolved optical grating (FROG) system (HR100 Optical pulse analyzer, Southern Photonics) and the pulse duration before compression was estimated to be approximately $2.62 \mathrm{ps}$, corresponding to a time-bandwidth product (TBP) of 2.23. The FROG traces shown in Fig 2(d) confirmed the large linear chirped of the output pulses.

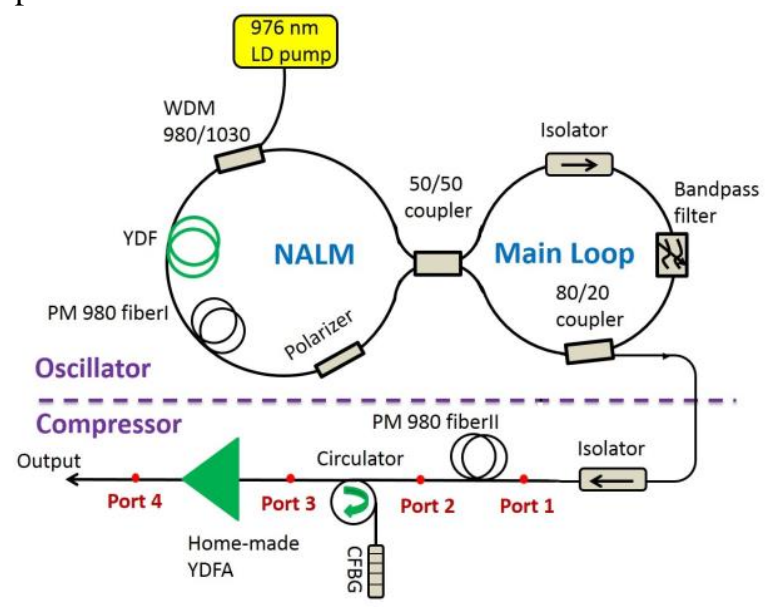

Fig.1. Schematic of all-PM figure of eight Yb fiber oscillator and compressor system. (YDF: Yb-doped fiber; CFBG: chirped fiber Bragg-grating; YDFA: Yb-doped fiber amplifier.)

\section{ALL-PM FIBER PULSE COMPRESSOR}

The linearly chirped pulses can be recompressed back to transform-limited pulses using a dispersion compensating device with a reversed chirp characteristic. Here we used an all-PM fiber compressor as depicted in the Fig.1 to maintain a stable all-fiber configuration of the laser system. Before de-chirping the highly positive-chirped pulses, tens of meters PM-980 fibers were incorporated for broadening the pulse, then the broadened pulse was launched into a PM chirped fiber Bragg grating (CFBG, reflection bandwidth of $\sim 20 \mathrm{~nm}$; chromatic dispersion of $2.85 \mathrm{ps} / \mathrm{nm}$, centered at $1030 \mathrm{~nm}$ ) through a PM optical circulator. The insertion loss of the optical circulator and CFBG was compensated with a PM ytterbium-doped fiber amplifier (YDFA). The YDFA consisted of $1.5 \mathrm{~m}$ highly-doped $\mathrm{Yb}$ fiber (core diameter: $6 \mu \mathrm{m}$, 
PM-YSF-LO, Nufern) spliced with a $\sim 10 \mathrm{~cm}$ PM-980 fiber pigtail for final output. In order to compensate the additional normal dispersion provided by the YDFA process, the pulse was intentionally over-compressed with the CFBG to give a negative chirped. By carefully adjusting the YDFA's pump level and optimizing the length of PM-980 fiber, transform limited pulses with a pulse width of $\sim 700$ fs was achieved (30 $\mathrm{m}$ PM-980 fiber, YDFA pump current of $50 \mathrm{~mW}$ ) with results as shown in the Fig. 3(a) and 3(b). Since the B-integral is small, there is negligible nonlinear effects in the amplification process in this proposed system. Output characteristics at different ports are summarized in Fig. 3(c). Apart from a higher noise background of port 4 due to the YDFA amplified spontaneous noise, the spectral shapes at each stage were kept relative constant with minimal spectral distortion, see Fig. 3(d). Due to the completely all-PM-fiber-integrated configuration, a remarkable long-term stability is expected.
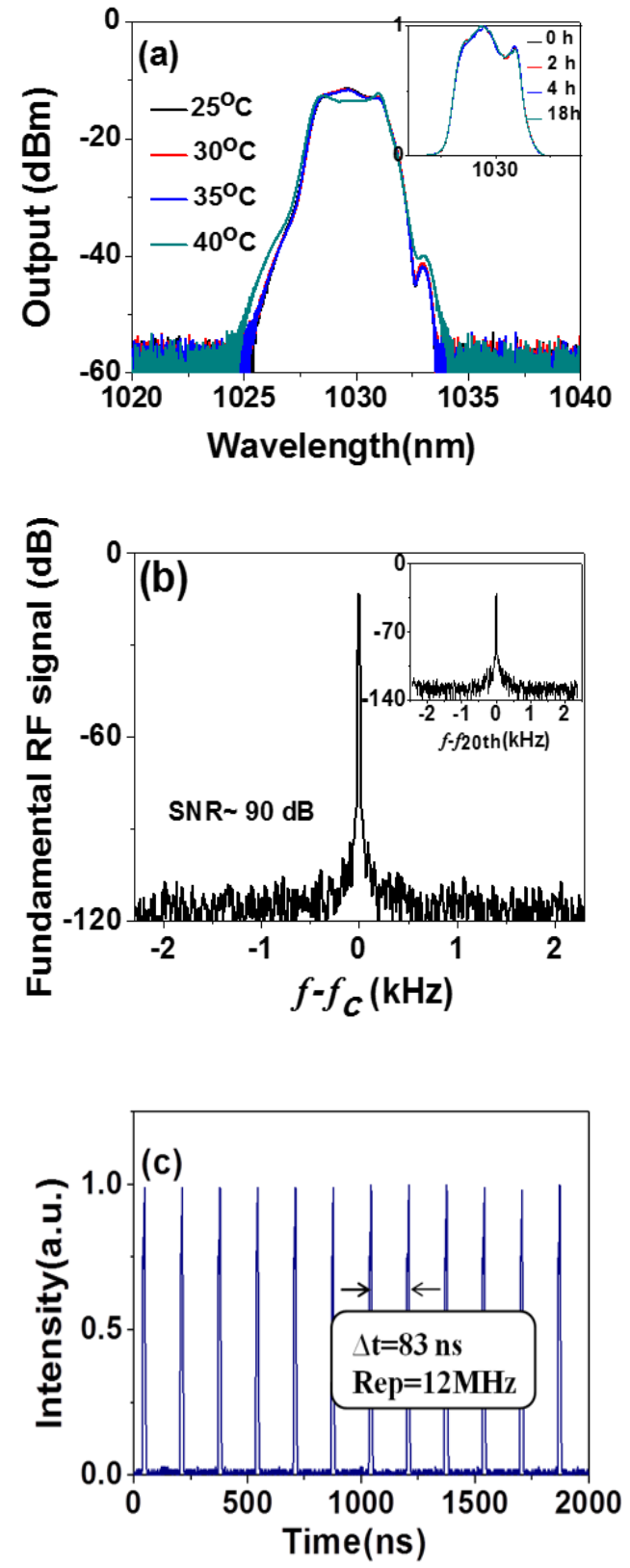

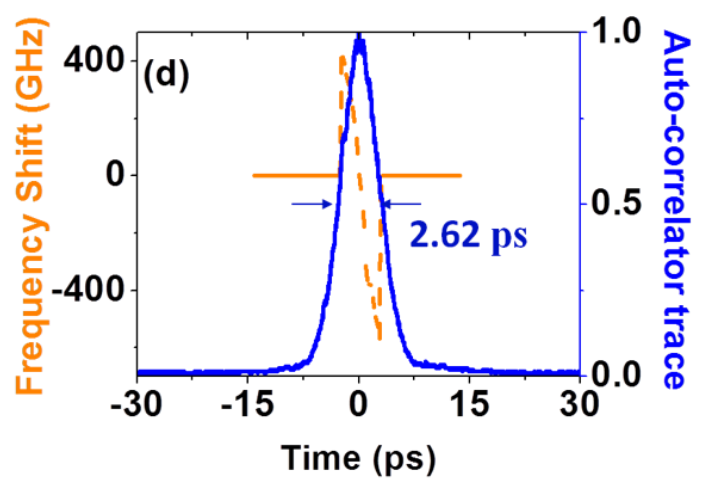

Fig.2. Output characteristics of all-PM figure of eight $\mathrm{Yb}$ fiber oscillator. Inset: Linear output spectra for long-term operation. (a) Output spectrum measured in different environment temperatures. (b) Fundamental RF signal. Inset: $20^{\text {th }}$-order frequency of $240 \mathrm{MHz}$ RF signal (c) Pulse train. (d) Autocorrelation trace and chirp line of pre-compression pulses.
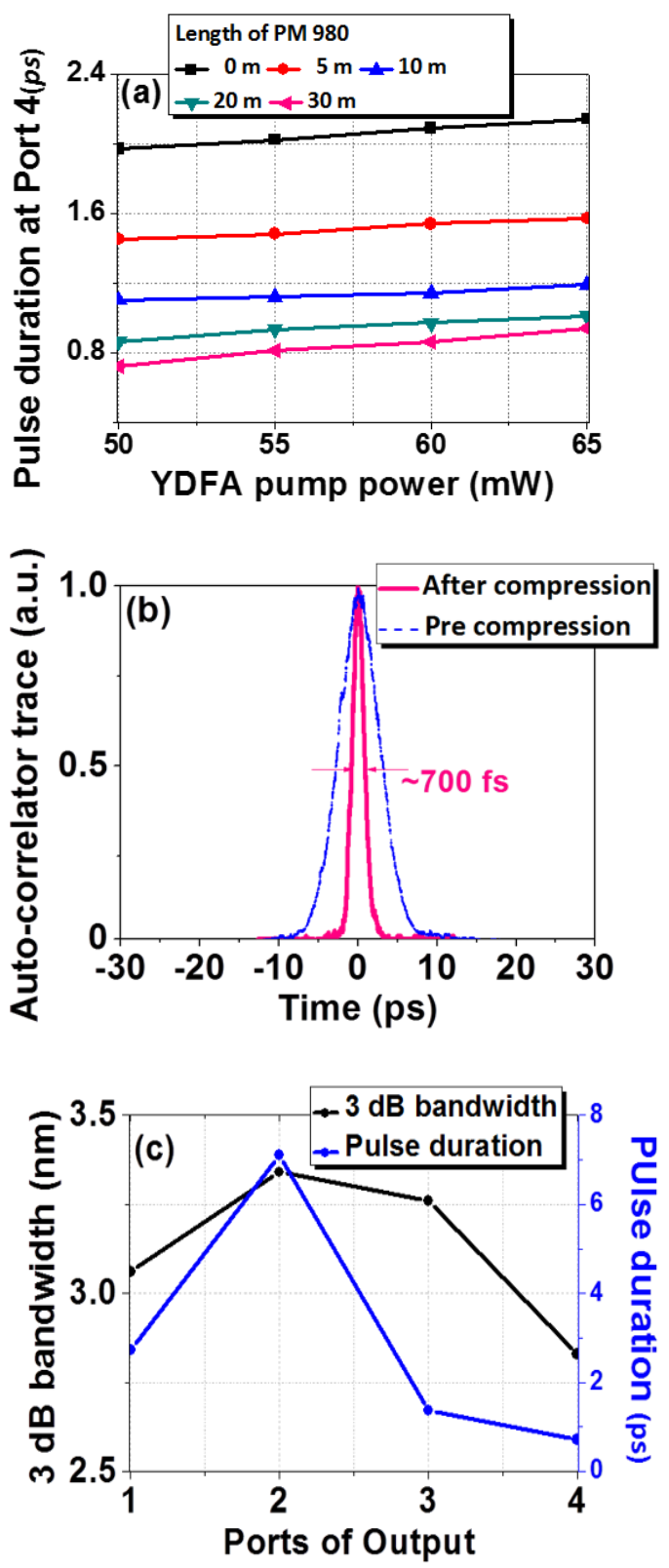


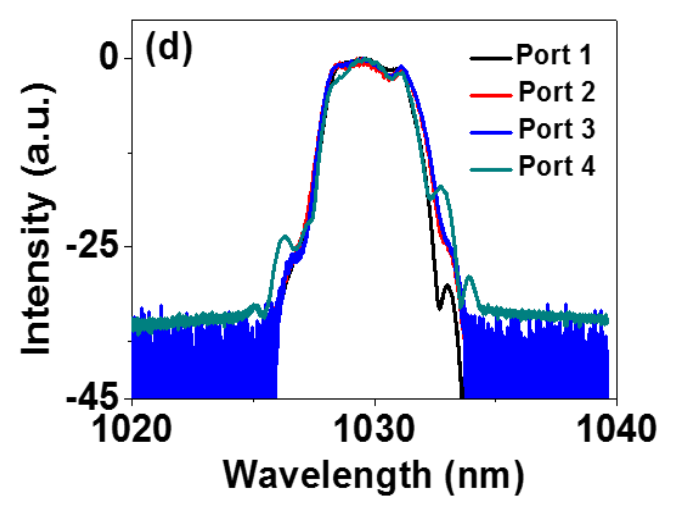

Fig.3. (a) YDFA pump current versus pulse duration at port 4 under different lengths of PM 980. (b) Auto-correlation traces of pre-compression and after compression. (c) Output characteristics of $3 \mathrm{~dB}$ bandwidth and pulse duration at different ports. (d) Normalized spectrum observed at the different output ports.

\section{DISCUSSION AND CONCLUSION}

We have proposed and demonstrated a simplified, compact, all-normal-dispersion, all-polarization-maintaining ultrafast fiber laser operating at $1030 \mathrm{~nm}$. The linearly-chirped output pulse was compressible to its transform-limited pulse duration of $700 \mathrm{fs}$ using an all-PM-fiber pulse compressor, with $0.45 \mathrm{~nJ}$ pulse energy and $12 \mathrm{MHz}$ repetition rate. Since NALM was employed as the mode-locker in this laser design, additional degree of freedom was possible for manipulating the output pulse characteristics. Higher pulse energy could be expected by carefully optimizing the NALM pulse and laser cavity design, such as the coupling ratio of interference coupler and the length of PM-980 fiber. Simple and compact all-PM-fiber laser systems such as the one presented here can provide practical solution for industrial and commercial products where system stability and robustness are indispensable.

\section{REFERENCES}

[1]. X. Zhao, G. Hu, B. Zhao, C. Li, Y. Pan, Y. Liu, T. Yasui, and Z. Zheng, "Picometer-resolution dual-comb spectroscopy with a free-running fiber laser," Opt. Express 24, 21833-21845 (2016).

[2]. H. Lim, Y. Jiang, Y. Wang, Y-C. Huang, Z. Chen, and F. W. Wise, "Ultrahigh-resolution optical coherence tomography with a fiber laser source at 1 um," Opt. Lett. 30, 1171-1173 (2005).

[3]. Y. Jeong, J. K. Sahu, D. N. Payne, and J. Nilsson, "Ytterbium-doped large-core fiber laser with $1.36 \mathrm{~kW}$ continuous-wave output power," Opt. Express 12, 6088-6092 (2004).

[4]. H. Lim, F. O. Ilday, and F. W. Wise, "Femtosecond ytterbium fiber laser with photonic crystal fiber for dispersion control," Opt. Express 10, 1497-1502 (2002).

[5]. R. Herda, S. Kivisto, O. G. Okhotnikov, A. F. Kosolapov, A. E. Levchenko, S. L. Semjonov, and E. M. Dianov, "Environmentally stable mode-locked fiber laser with dispersion compensation by Index-guided photonic crystal fiber," IEEE. PHOTONIC TECH L 20, 217-219 (2008).

[6]. Z. X. Zhang, C. Senel, R. Hamid, and F. O. Ilday, "Sub-50 fs Yb-doped laser with anomalous-dispersion photonic crystal fiber," Opt. Lett. 38, 956-958 (2013)

[7]. K. Tamura, E. P. Ippen, H. A. Haus, and L. E. Nelson, "77-fs pulse generation from a stretched-pulse mode-locked all-fiber ring laser," Opt. Lett. 18, 1080-1082 (1993).

[8]. F. O. Ilday, J. R. Buckley, W. G. Clark, and F. W. Wise, " Self-Similar Evolution of Parabolic Pulses in a Laser, " Phys. Rev. Lett. 92, 213902-1-213902-4 (2004).

[9]. A. Chong, J. Buckley, W. Renninger, and F. W. Wise“All-normal-dispersion femtosecond fiber laser," Opt. Express 14, 10095-10100 (2006).
[10]. C. K. Nielsen, B. Ortac, T. Schrebier, J.Limpert, R. Hohmuth, W. Richter, and A. Tunnermann, "Self-starting self-similar all-polarization maintaining Yb-doped fiber laser," Opt. Express 13, 9346-9351 (2005).

[11]. I. Hartl, G. Imeshev, L. Dong, G. C. Cho and M. E. Fermann, "Ultra-compact dispersion compensated femtosecond fiber oscillators and amplifiers," CLEO, Baltimore, paper CThG1, May 2005.

[12]. K. Viskontas, K. Regelskis, and N. Rusteika, "Slow and fast optical degradation of the SESAM for fiber laser mode-locking at $1 \mu \mathrm{m}$," Lithuanian Journal of Physics, 54, 127-135 (2014).

[13]. K. Kieu, W. H. Renninger, A. Chong, and F. W. Wise, "Sub- 100fs pulses at watt-level powers from a dissipative-soliton fiber laser," Opt. Lett. 34, 593-595 (2009).

[14]. D. S. Kharenko, E. V. Podivilov, A. A. Apolonski, and S. A. Babin, "20 nJ 200 fs all-fiber highly chirped dissipative soliton oscillator," Opt. Lett. 37, 4104-4106 (2012)

[15]. J. Szczepannek, T. M. Kardas, C. Radzewicz, and Y.Stepanenko, "Ultrafast laser mode-locked using nonlinear polarization evolution in polarization maintaining fibers," Opt. Lett. 42, 575-578 (2017).

[16]. N. J. Doran and D. Wood, "Nonlinear-optical loop mirror," Opt. Lett. 13, 56-58 (1988).

[17]. M. E. Fermann, F. Haberl, M. Hofer, and H. Hochreiter, "Nonlinear amplifying loop mirror," Opt. Lett. 15, $752-754$ (1990).

[18]. Y. S. Fedotov, A. V. Ivanenko, S. M. Kobtsev, and S. V. Smirnov, "High average power mode-locked figure-eight $\mathrm{Yb}$ fibre master oscillator," Opt. Express 22, 31379-31386 (2014).

[19]. F. Ö. Ilday, F. W. Wise, and T. Sosnowski, "High-energy femtosecond stretched-pulse fiber laser with a nonlinear optical loop mirror," Opt. Lett, 27, 1531-1533 (2002).

[20]. C. Aguergaray, N. G. R. Broderick, M. Erkintalo, J. S. Y.Chen, and V. Kruglov, "Mode-locked femtosecond all-normal all-PM Yb-doped fiber laser using a nonlinear amplifying loop mirror," Opt. Express 20,10545-10551 (2012).

[21]. M. Erkintalo, C. Aguergaray, A. Runge, and N. G. R. Broderick, "Environmentally stable all-PM all-fiber giant chirp oscillator," Opt. Express 20, 22669-22674 (2012).

[22]. J. Szczepanek, T. M. Kardas, M. Michalska, C. Radzewicz, and Y. Stepanenko, "Simple all-PM-fiber laser mode-locked with a nonlinear loop mirror," Opt. Lett. 40, 3500-3503 (2015).

[23]. P. Bowen, H. Singh, A. Runge, R. Provo, and N. G. R. Broderick, “,” Optics. Communications 364, 181-184 (2016).

[24]. S. M. Ou, G. Y. Liu, L. Guo, Z. G. Zhang and Q. M. Zhang, "870 fs, 448 $\mathrm{kHz}$ pulses from an all-polarization-maintaining $\mathrm{Yb}$-doped fiber laser with a nonlinear amplifying loop mirror, "Applied Optics,57,5068-5071 (2018).

[25]. Y. Yu, H. Teng, H. B. Wang, L. N. Wang, J. F. Zhu, S. B. Fang, G. Q. Chang, J. L. Wang and Z. Y. Wei, "Highly-stable mode-locked PM Yb-fiber laser with $10 \mathrm{~nJ}$ in 93 -fs at $6 \mathrm{MHz}$ using NALM, "Opt. Express 26, 10428-10434 (2018).

[26]. B. Xu, A. Martinez, S. Y. Set, C. S. Goh and S. Yamashita, "A net normal dispersion all-fiber laser using a hybrid mode-locking mechanism, " Laser Physics Letters, 11, 025101 (2014).

[27]. B. Xu, A. Martinez, S. Y. Set, C. S. Goh and S. Yamashita, " Polarization Maintaining, Nanotube-based mode-locked lasing from Figure of Eight Fiber Laser, " IEEE. PHOTONIC TECH L 26, 180-182 (2014). 